\title{
Cleaner production of salt-tolerance vegetable in coastal saline soils using reclaimed water irrigation: Observations from alleviated accumulation of endocrine disrupting chemicals and environmental burden
}

\author{
Jian $\mathrm{Lu}^{\mathrm{a}, \mathrm{b}}$, Jun $\mathrm{Wu}^{\mathrm{c}, \mathrm{d},{ }^{*}, \text { Cui Zhang }}{ }^{\mathrm{a}}$ \\ a CAS Key Laboratory of Coastal Environmental Processes and Ecological Remediation, Yantai Institute of Coastal Zone Research (YIC), Chinese Academy of \\ Sciences (CAS), Shandong Key Laboratory of Coastal Environmental Processes, YICCAS, Yantai, Shandong, 264003, China \\ ${ }^{\mathrm{b}}$ Center for Ocean Mega-Science, Chinese Academy of Sciences, 7 Nanhai Road, Qingdao, Shandong, 266071, China \\ c Yantai Research Institute and Graduate School, Harbin Engineering University, Yantai, Shandong, 265501, China \\ ${ }^{\mathrm{d}}$ College of Materials and Chemical Engineering, Harbin Engineering University, Harbin, Heilongiang, 150001, China
}

\section{A R T I C L E I N F O}

\section{Article history:}

Received 9 December 2020

Received in revised form

28 February 2021

Accepted 12 March 2021

Available online 17 March 2021

Handling editor: Cecilia Maria Villas Bôas de Almeida

\section{Keywords:}

Saline soil utilization

M. crystallinum

Endocrine disrupting chemicals

Reclaimed water irrigation

Coastal zone

\begin{abstract}
A B S T R A C T
It is important to investigate the feasibility of reclaimed water irrigation for fulfilling cleaner production of salt-tolerant vegetables in coastal saline soils which widely distribute in the world. Reclaimed water irrigation might cause the accumulation of endocrine disrupting chemicals in crops although it is a good approach for alleviating water resource shortage. This study firstly investigated the possible cleaner production and benefits of cultivating Mesembryanthemum crystallinum (M. crystallinum) in coastal saline soil irrigated by simulated reclaimed water. Concentrations of target pollutants including bisphenol A (BPA) and nonylphenol (NP) in root of $M$. crystallinum were higher than those in stem and leaf while soil salinity could increase the concentrations of BPA/NP in root and decrease those in leaf and stem. The highest concentration of BPA/NP in root of $M$. crystallinum growing in soil with salt content of $7.2 \%$ reached $29.28 / 243.47 \mu \mathrm{g} / \mathrm{kg}$. BPA mainly accumulated in leaf of $M$. crystallinum with the highest amount of $621.93 \mathrm{ng}$ under non-saline conditions while NP mainly accumulated in root with the highest amount of $1114.57 \mathrm{ng}$ under saline conditions. The hazard quotients sharply decreased by $>50 \%$ when the soil salinity increased from $1.1 \%$ to $7.2 \%$. Non-cancer health risks of BPA and NP in M. crystallinum were acceptable for all human groups while the daily intake of BPA/NP was much lower than the corresponding tolerant daily intake. Life cycle assessment illustrated that M. crystallinum cultivation in soil with salt content of $1.1 \%$ posed the lowest environmental burden. Salt-tolerant vegetables such as M. crystallinum would be recommended to be cultivated in low-salinity saline soils using reclaimed water irrigation due to lower pollutant accumulation and environmental burden to achieve the goals of cleaner vegetable production. These findings provide new insight on the promising potential of reclaimed water irrigation and crop cultivation in saline soils in coastal regions.
\end{abstract}

() 2021 Elsevier Ltd. All rights reserved.

\section{Introduction}

Cleaner production has become an important strategy for sustainable development of various fields such as biodiesel production (Athar and Zaidi, 2020), plastic granule production (Cascone et al., 2020), and food production (Santos et al., 2018). Humans have

\footnotetext{
* Corresponding author. Yantai Research Institute and Graduate School, Harbin Engineering University, Yantai, Shandong, 265501, China.

E-mail address: wujunlisa@163.com (J. Wu).
}

faced a series of problems including extensive exploration of resources, release of massive wastes, and environmental pollution so that cleaner production has become an inevitable alternative for the traditional production activities to support the sustainable human society. Many techniques including green engineering (Van Loy et al., 2018), waste reuse/recycle (Guerra et al., 2020), and pollutant reduction (Ahmadisharaf and Benham, 2020) have been established to achieve the goals of cleaner production. Efforts on cleaner agricultural production are still needed due to the current limited studies. 
Saline soils have widely distributed in global arid and semi-arid areas (Wang et al., 2020) to cause waste of land resource due to low productivity. Current utilization of saline soils includes land application after amelioration and cultivation of salt-tolerance plants (Chiconato et al., 2019). Salt-tolerance plant cultivation is perfect for salt-affected soil usage due to both economic benefit and soil amelioration effect (Chiconato et al., 2019). Study on cultivation of salt-tolerance economic plants in salt-affected soils is not enough yet to obtain comprehensive information on its cleaner production. It is feasible to cultivate the salt-tolerance vegetables and crops in salt-affected soils using reclaimed water which contains enough nutrients for plant growth to reach goals of both saving water resource and utilizing the saline lands. Vegetable cultivation in salt-affected soils might be an effective approach for cleaner food production.

The Mesembryanthemum crystallinum (M. crystallinum), also called ice plant, is a halophyte originating from Africa (Atzori et al., 2017) to be introduced into China as a kind of popular leafy vegetables for years. Current research work on M. crystallinum still mainly focuses on its growth conditions and physiologicalbiochemical properties although $M$. crystallinum has shown good tolerance to stress caused by salt and some heavy metals (Atzori et al., 2017). M. crystallinum might be a perfect candidate for testing feasibility of reclaimed water irrigation in salt-affected soils. The reclaimed water is an essential water resource that was widely used for irrigation due to the shortage of freshwater resources in coastal regions (Lu et al., 2020a). Reclaimed water could also be an important pollution source of emerging contaminants such as endocrine disrupting chemicals (EDCs) for crops (Lu et al., 2015) and water (Lu et al., 2020a). Current investigations on reclaimed water exert efforts on discussing the potential effect of its application including irrigation and recharge (Lu et al., 2020b). Rare information is currently available on accumulation of EDCs in salttolerance plant such as $M$. crystallinum growing in saline soils irrigated by reclaimed water as well as the potential environmental assessment of this process.

EDCs widely exist in different matrices to pose potential risks to ecosystem and human health (Lu et al., 2020b). As a kind of emerging contaminants, EDCs have attracted more attention due to its adverse effect with low concentration and pseudo-persistence (Giulivo et al., 2016). Many substances including natural hormones and artificial chemicals such as heavy metals, phenolic chemicals, pesticides, and persistent organic pollutants (Futran Fuhrman et al., 2015) have exhibited endocrine-disrupting features. Among artificial EDCs, bisphenol A (BPA) and nonylphenol (NP) deserve more attention since they generally exist in environments with relatively high concentrations and wide existence (Hermabessiere et al., 2017). BPA and NP have been frequently detected in different matrices (Santonicola et al., 2020) to exert potential threat to humans. They have been observed to accumulate in vegetables under reclaimed water irrigation which is generally regarded as a good way for solving problem of water resource shortage (Dodgen et al., 2013). It is necessary to discuss the food safety with reclaimed water irrigation, especially the possible accumulation of pollutants including EDCs during salt-tolerance plant cultivation in salt-affected soils using reclaimed water owing to the scarce relevant information. Reduction on accumulation of EDCs in plants also needs further investigation to meet requirements of cleaner agricultural production.

Anthropogenic activities will cause environmental burden to the ecosystem and health risks to humans so that many methods have been developed to evaluate the environmental impact of these activities (Wen et al., 2019) as well as to establish novel techniques to reduce various pollutants. Health risk evaluation is useful to quantify the potential risks of pollutants to humans (Lu et al., 2020b). Life cycle assessment (LCA) is a good approach for environmental impact evaluation by considering the whole processes "from cradle to grave" (Gunady et al., 2012). LCA is also a good tool for assessing the potential impact of cultivation of M. crystallinum in saline soils under the conditions of reclaimed water irrigation. LCA results will provide useful information on cleaner agricultural production in saline soils.

This study will investigate the effect of soil salinity on accumulation of typical phenolic EDCs in M. crystallinum irrigated by simulated reclaimed water. The LCA of $M$. crystallinum cultivated in coastal saline soils was also conducted. The final goal is to provide initial information on risk alleviation of reclaimed water irrigation in coastal zone by considering the potential environmental impact on saline soil utilization. This study firstly reported the possibility and feasibility of reclaimed water irrigation in saline soils for $M$. crystallinum cleaner production. The findings of this work will provide useful information on utilization of saline soils, reclaimed water application, and vegetable cleaner production.

\section{Materials and methods}

\subsection{Standards, reagents, and chemicals}

Standards including BPA (purity $>99 \%$ ), NP (4-n-NP, purity $>98 \%$ ), and surrogate ( $\beta$-estradiol 17 -acetate with purity $\geq 99 \%$ ) were purchased from Sigma-Aldrich (St. Louis MO, USA). Bisphenol A-D16 (BPA-D16, 98 atom\% D) was used as isotope dilution standard (IDS) and obtained from Sigma-Aldrich (St. Louis MO, USA). Trimethylchlorosilane (TMCS, purity> 98\%) and N,O-bis(trimethylsilyl) trifluoroacetamide (BSTFA, purity $>98 \%$ ) were obtained from Alfa Aesar (Ward Hill, MA). Solvents including acetonitrile, acetone, and methylene chloride with HPLC grade were purchased from Merck (Germany). Standards were individually dissolved in acetonitrile to make stock solution with concentrations of $1 \mathrm{~g} / \mathrm{L}$ which was stored at $4{ }^{\circ} \mathrm{C}$ before use. The simulated reclaimed water was made by diluting BPA/NP stock solution using deionized water to reach irrigation concentration of $20 \mu \mathrm{g} / \mathrm{L}$ which was consistent with previous reports (Lu et al., 2020a).

\subsection{Plant materials, coastal saline soils, and experiments}

The experiment was performed by following the flowchart (Fig. 1). M. crystallinum and soils were carefully prepared. The cultivation experiments were conducted by using simulated reclaimed water containing BPA/NP. Different tissue parts were weighed and analyzed. Health risk evaluation and life cycle assessment were also conducted to determine the various effects of soil salinity.

The $M$. crystallinum seeds were purchased from a seed company (Weifang, China). Seeds were washed and sown on organic substrate at $25{ }^{\circ} \mathrm{C}$ with relative humidity of $60 \%-70 \%$ in the dark for 7 days. $M$. crystallinum seedlings after 2 -week germination were transplanted in experimental pots with different soils. Simulated reclaimed water was daily irrigated for these seedlings. Coastal soils were collected from the Yellow River Delta. Soil with salinity of $1.1 \%$ o $2.4 \%$ o $7.2 \%$ was used as the representative soils and denoted as SAS1/SAS2/SAS3. Control soil samples were collected from a garden of Yantai and denoted as SCK. The analysis methods of soil physio-chemical properties referred to Wang et al. (2020). The detailed physio-chemical properties of target soils were listed in Table 1. BPA and NP were not detected in all soils.

Base fertilizer was firstly added into different soils at addition amount of $6 \mathrm{mg} / \mathrm{cm}^{2}$ to provide enough nutrient for growth of $M$. crystallinum. Real reclaimed water contains different components to possibly exert complex effect on accumulation of EDCs in 


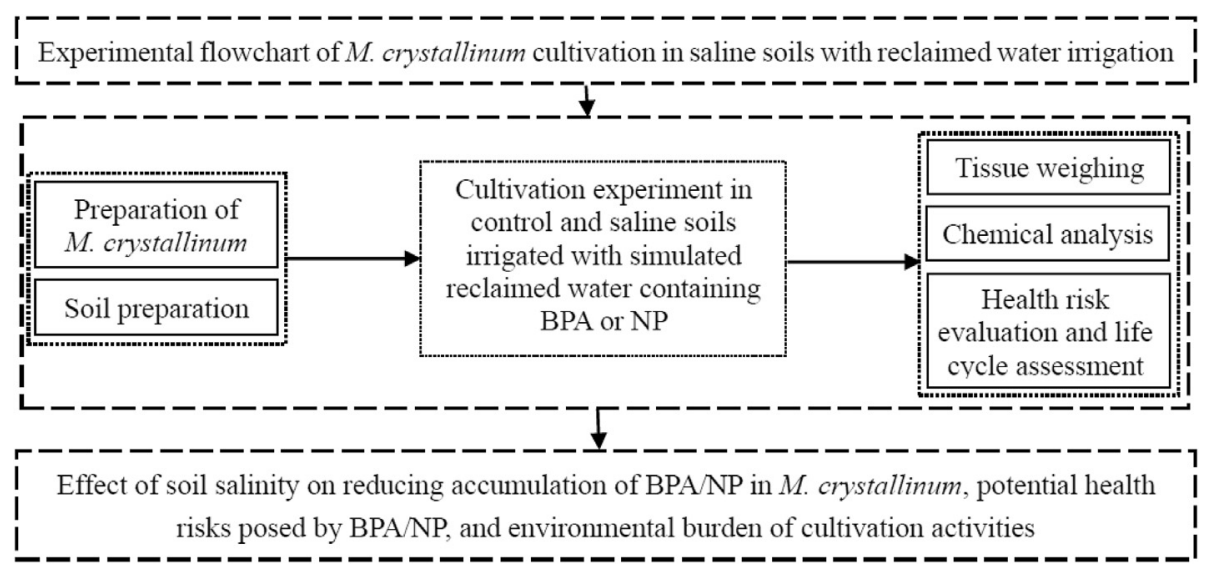

Fig. 1. Flowchart of the experimental design for this study.

Table 1

Basic properties of the common soil and salt-affected soils.

\begin{tabular}{|c|c|c|c|c|c|c|c|c|c|}
\hline Soil & Soil salinity & Salt content $(\% 0)$ & $\mathrm{pH}$ & $\mathrm{EC}(\mathrm{dS} / \mathrm{m})$ & Organic matter (g/kg) & $\mathrm{CEC}(\mathrm{cmol} / \mathrm{kg})$ & Sand & Silt & Clay \\
\hline & & & & & & & \multicolumn{3}{|c|}{$---(\%)---$} \\
\hline SCK & Non-saline & 0.5 & 7.50 & 0.08 & 22.13 & 8.27 & 20.49 & 37.73 & 41.78 \\
\hline SAS1 & Very slightly saline & 1.1 & 7.62 & 0.43 & 20.21 & 8.98 & 22.72 & 32.89 & 44.39 \\
\hline SAS2 & Slightly saline & 2.4 & 7.55 & 0.86 & 13.02 & 16.12 & 12.78 & 38.65 & 48.57 \\
\hline SAS3 & Moderately saline & 7.2 & 7.52 & 3.01 & 19.78 & 6.08 & 21.49 & 39.26 & 39.25 \\
\hline
\end{tabular}

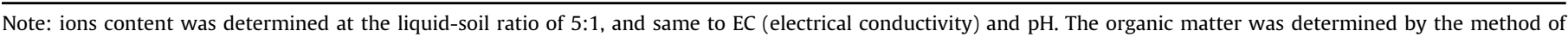
potassium dichromate heating.

M. crystallinum. The simulated reclaimed water was used in this study to simplify the experiment and eliminate the impact of the other interference factors. Simulated reclaimed water containing BPA or NP was daily added to experimental pots by root irrigation. Pots were placed in a chamber for growth at $25 \pm 1{ }^{\circ} \mathrm{C}$ with relative humidity of $60 \%-70 \%$. Light condition was set with 16 -h photoperiod and 8-h night. Treated plants were harvested after 30-day irrigation and ready for analysis. Each plant after harvest was rinsed by deionized water and dissected into root, stem, and leaf parts. Fresh weight of root/stem/leaf for each plant was recorded. All plant samples were stored at $-20{ }^{\circ} \mathrm{C}$ until extraction. Each assay was performed in triplicate.

\subsection{Sample preparation and instrumental analysis}

The plant extraction procedure referred to the previous report (Lu et al., 2015). In brief, 5 g of plant samples mixed by $100 \mathrm{~mL}$ acetone were added by IDS and surrogate at spiking amount of $50 \mu \mathrm{g} / \mathrm{kg}$. The plant samples were homogenized by a blender, ultrasonically extracted for $5 \mathrm{~min}$, filtered, and evaporated to dryness. The dried samples were re-dissolved in acetonitrile and ready for acid hydrolysis. The hydrolyzed samples were extracted by methylene chloride. The extracts were dried and derivatization using BSTFA (99\%)-TMCS (1\%). The derivatized extracts were dried by high-pure nitrogen and dissolved in $100 \mu \mathrm{L}$ of hexane for instrumental analysis.

The instrumental analysis referred to Lu et al. (2020b). In summary, the target EDCs were analyzed by GC-MS (Agilent 7820A GC system with M7 single quadrupole MS system) equipped with a $30 \mathrm{~m}$ DB-5MS column. The carrier gas was helium (purity $>99.999 \%$ ) at $1.0 \mathrm{~mL} / \mathrm{min}$ and the injection volume was $1 \mu \mathrm{L}$ at splitless mode. Temperature program was set as the followings: column oven $\left(50^{\circ} \mathrm{C}\right)$ kept for $1 \mathrm{~min}$, increased to $120^{\circ} \mathrm{C}$ at $20^{\circ} \mathrm{C} /$ min and hold for $3 \mathrm{~min}$, and increased to $280^{\circ} \mathrm{C}$ at $8^{\circ} \mathrm{C} / \mathrm{min}$ to keep for $8 \mathrm{~min}$. Retention time and selective ions were together used to confirm BPA and NP (Lu et al., 2020b). The limit of detection (LOD) for BPA/NP was $0.13 / 0.21 \mu \mathrm{g} / \mathrm{kg}$.

Two spiked plant samples (M. crystallinum bought from the local market without detected BPA and NP) with spiking concentration of $20 \mu \mathrm{g} / \mathrm{kg}$ were analyzed for every 10 samples. Recoveries of spiking plant samples were in the range of $(93 \%-99 \%) /(91 \%-102 \%)$ for BPA/NP with relative standard deviation (RSD) less than $6 \%$ to guarantee quality control of analysis.

\subsection{Health risks assessment and LCA}

Principal component analysis (PCA) was performed on OriginPro 2019 (OriginLab, USA). Interaction network analysis was conducted on Cytoscape 3.7.2 (The Cytoscape Consoritum, USA) with absolute value of Spearman coefficient greater than 0.6 and $p<0.05$. Correlation analysis with significance at $p<0.05$ was performed by SPSS 19 (IBM, USA).

Health risks posed by target EDCs were evaluated by hazard quotients $(H Q)$. The detailed calculation referred to Wen et al. (2019) with some modifications.

$H Q=\frac{C \times I R \times E F \times E D}{B W \times A T \times R f D}$

where $R f D$ represents the reference dose through oral exposure route which is $6.00 \times 10^{-3} / 6.48 \times 10^{-2} \mathrm{mg} /(\mathrm{kg} \cdot$ day $)$ for BPA/NP; $A T$ stands for average lifespan; $B W$ means body weight; $C$ refers to concentration of BPA/NP in $M$. crystallinum; IR represents vegetable consumption rate; $E D$ refers to ingestion exposure duration; $E F$ refers to ingestion exposure frequency ( $E F=365$ days/yr). Vegetable consumption rate (IR) of different groups referred to previous reports of China (Li et al., 2017; Zheng et al., 2007) and other country (Rosário et al., 2018) with value of 231.5/292/245.5/344.3/ 
$356.7 \mathrm{~g} / \mathrm{d}$ for children/female teenagers/male teenagers/female adults/male adults. $A T$ value is set as 2190/6570/25550 days and $E D$ value is set as 6/18/70 years for children/teenagers/adults; $B W$ value is set as $19.73 / 47.64 / 51.24 / 55.18 / 63.29 \mathrm{~kg}$ for children/female teenagers/male teenagers/female adults/male adults. Threshold of HQ was set as 1 (Wen et al., 2019).

The LCA on M. crystallinum cultivation activities was performed by Simapro 7.1 (PRé Consultants, Netherland). The function unit was set as $1 \mathrm{~kg}$ of $M$. crystallinum. The life cycle inventories (LCI) mainly included irrigation and transportation. The LCI data were linked to the corresponding library of Simapro 7.1. Eco-indicator 99 (H) V2.06/Europe EI 99 H/A was employed to evaluate the potential impacts of $M$. crystallinum cultivation activities. Impact categories including climate change, ecotoxicity, acidification/eutrophication, fossil fuels, and land use were assessed. The detailed input/output/ method information referred to "Database manual" and "Introduction into LCA" of Simapro 7.1. LCA results on ice plant cultivation in saline soils using reclaimed water irrigation might be partially affected by site conditions which would influence some LCA inputs so that LCA of different regions is needed in the future.

\section{Results and discussion}

\subsection{Distribution and accumulation of BPA and NP in M. crystallinum}

The yield of $M$. crystallinum in SAS1/SAS2/SAS3 was $97.1 \% / 90.2 \% /$ $73.6 \%$ of that in SCK for BPA treatment while the plant yield in SAS1/ SAS2/SAS3 was $93.8 \% / 87.4 \% / 71.6 \%$ of that in SCK for NP treatment. Soil salinity of $1.1 \%$ or $2.4 \%$ did not significantly decrease the yield of M. crystallinum. Distribution of BPA and NP in M. crystallinum showed significant difference (Fig. 2). Concentrations of BPA and NP in root were higher than those in other parts of M. crystallinum growing in different soils, especially for NP. Soil salt could enhance the concentration of BPA and NP in root of M. crystallinum. Concentration of BPA in root of M. crystallinum growing in SAS3 with salt content of $7.2 \%$ reached $29.28 \mu \mathrm{g} / \mathrm{kg}$, almost 1.37 times that growing in SCK and 1.29/1.16 times that growing in SAS1/SAS2 (Fig. 2a). It was interesting that concentrations of NP in root of M. crystallinum were significantly higher than those of BPA, all higher than $200 \mu \mathrm{g} / \mathrm{kg}$ (Fig. 2b). Similarly, soil salt enhanced the concentration of NP in root of $M$. crystallinum. Concentration of NP in root of M. crystallinum growing in SAS3 reached $243.47 \mu \mathrm{g} / \mathrm{kg}$, almost 1.16 times that growing in SCK and 1.07/1.03 times that growing in SAS1/SAS2 (Fig. 2b). Different from root, concentration of BPA and NP in stem and leaf decreased with the salt content (Fig. 2). Concentrations of BPA and NP in stem were much lower than those in root and leaf. Concentrations of BPA and NP in different parts of $M$. crystallinum followed the order of root>leaf $>$ stem. Concentrations of BPA in leaf/stem were averagely $57.3 \% / 33.4 \%$ those in root while concentrations of NP in leaf/stem were averagely $4.1 \% / 1.2 \%$ those in root, illustrating significant distribution difference caused by chemical properties of target EDCs. Considering leaf and stem are the main edible tissues of vegetables, relatively low concentrations of target EDCs in stem and leaf might be good news for consumers and producers. Concentration of BPA and NP in stem or leaf of M. crystallinum growing in SAS3 was less than $50 \%$ of that growing in control soil. Salt stress showed the similar negative effect on distribution of BPA and NP in stem and leaf (Fig. 2). Higher salinity significantly decreased the concentrations of BPA and NP in stem and leaf of M. crystallinum. Similar trends of other pollutants such as heavy metals in plants under salt stress were also reported (Fritiof et al., 2005). Concentration and accumulation amount of copper and cadmium in both Elodea and Potamogeton decreased with increased salinity (Fritiof et al., 2005).
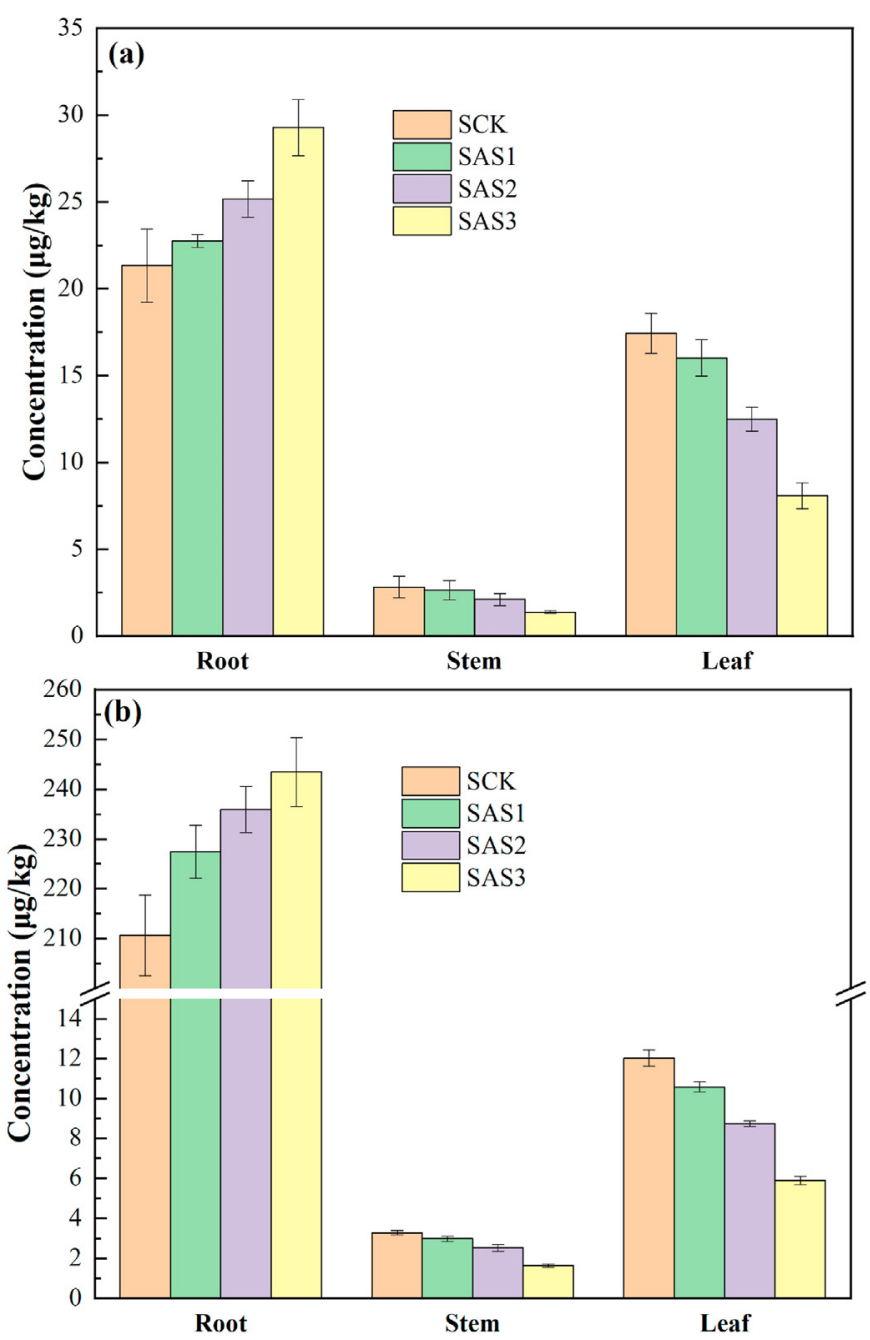

Fig. 2. Distribution of BPA (a) and NP in different parts of $M$. crystallinum. SCK refers to control soil; SAS1/SAS2/SAS3 refers to salt-affected soil with salinity of $1.1 \%$ o $2.4 \%$ o/ $7.2 \%$.

It was interesting that increased salinity could only decrease the concentration of zinc in cucumber while the other metals such as lead and copper showed the opposite variations (Taghipour and Jalali, 2019). Effects of soil salinity on accumulation of pollutants in the plants might be related to various factors such as physiochemical properties of pollutants and binding pathways of pollutants in the plants. Reclaimed water irrigation has been widely used for agriculture in many areas such as coastal zone (Lu et al., 2020a) and urban parks (Zalacáin et al., 2019). Reclaimed water irrigation can be an important trial for cleaner production of crops in saline soils due to rich nutrients in reclaimed water. However, accumulation of salt and pollutants in plants was frequently observed (Zalacáin et al., 2019), which negatively affected the further application of reclaimed water irrigation to some extent. Soil salt could inhibit the accumulation of BPA and NP in leaf and stem of M. crystallinum, which provided good news on possible cleaner production of salt-tolerant vegetables in saline soils.

The BPA and NP in M. crystallinum were mainly influenced by factor 1 which was the salinity of the soil according to the PCA analysis (Fig. 3). The control group was quite different from the groups under different salinity treatments. The SAS1 group was closer to SCK group than the other saline groups, illustrating that salinity exposure significantly influenced the uptake of EDCs in 

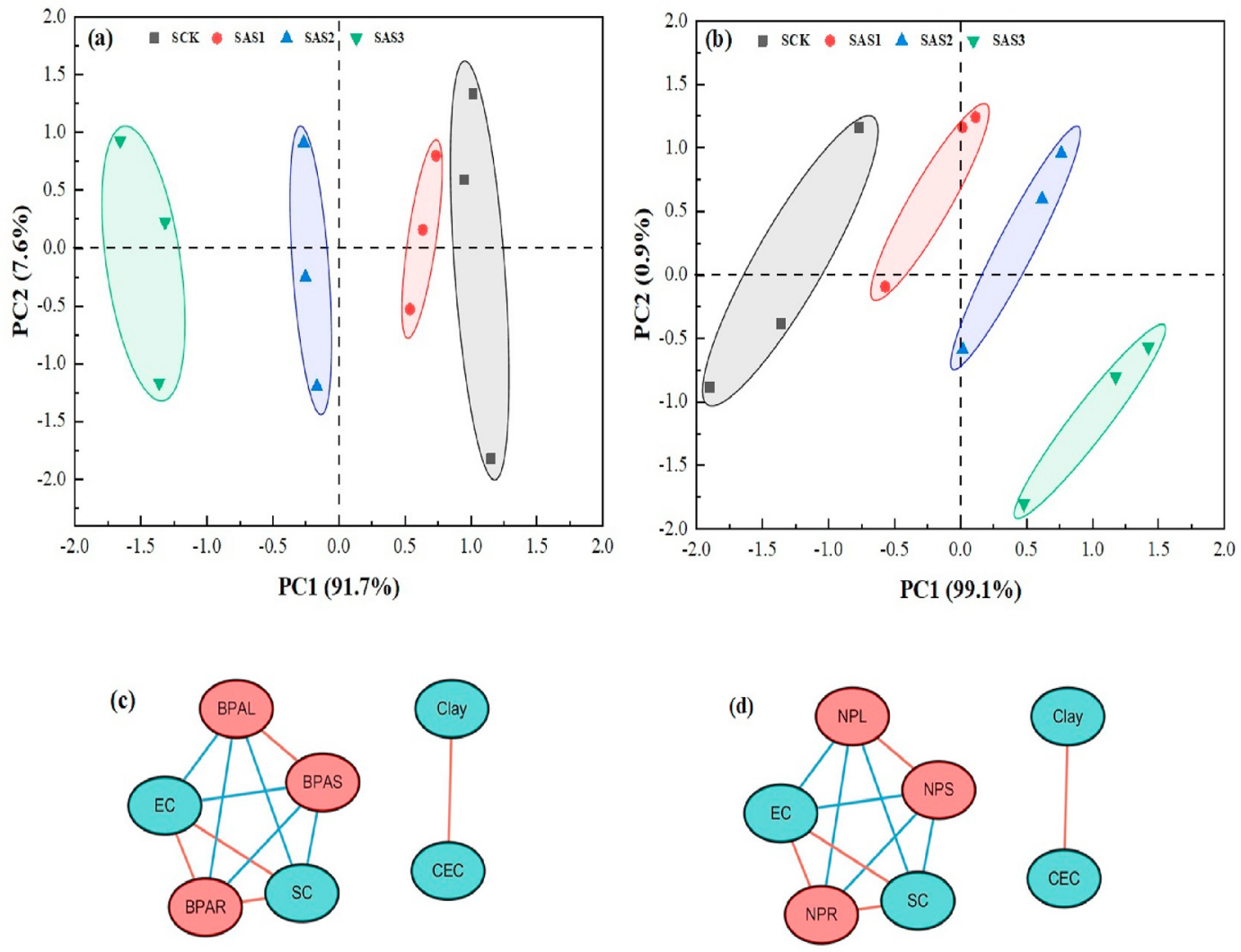

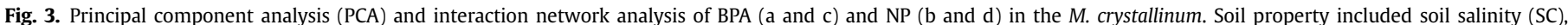

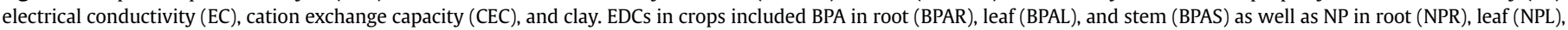

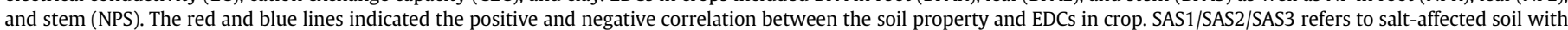
salinity of $1.1 \% \circ / 2.4 \% 0 / 7.2 \%$.

crops. The distance between the control group and salinity treatment group increased with the soil salinity (Fig. 3a and b). Additionally, the concentration of BPA and NP in root had strong positive relationship with the soil EC and salinity while those in the shoot had strong negative relationship with the soil EC and salinity (Fig. 3c and d), confirming that the uptake of the EDCs in shoot could be inhibited by the soil salinity. Relatively lower concentrations of target EDCs in shoot could decrease the potential health risk of consuming $M$. crystallinum. Relatively high levels of soluble salt in soils could increase osmotic stress to affect the water uptake and imbalanced accumulation of ions in the plants (Minhas et al., 2020), which did harm to the plant growth but possibly had good effect on controlling accumulation of pollutants. Ions in reclaimed water with soil soluble salts together might participate in the competition of NP and BPA in the plant to further inhibit the transport of target EDCs from root to shoot. Reclaimed water irrigation in coastal saline soils showed good effect not only on water resource conservation but also on controlling the accumulation of pollutants, exhibiting prospective potential in cleaner vegetable production.

The BPA mainly accumulated in leaf of M. crystallinum while NP mainly accumulated in root (Fig. 4). Accumulation amount of BPA in root reached the highest with $115.74 \mathrm{ng}$ for $M$. crystallinum growing in SAS2 with salt content of $2.4 \%$ while accumulation amount of NP in root reached the highest (1114.57 $\mathrm{ng}$ ) for the plant growing in SAS1 with salt content of $1.1 \%$ (Fig. 4). Accumulation of BPA and NP in leaf and stem decreased with salt content of soils. Accumulation amount of BPA in stem of plant growing in SAS1/SAS2/SAS3 was $86.0 \% / 49.9 \% / 23.4 \%$ that of plant growing in control soil while accumulation amount of BPA in leaf of plant growing in SAS1/SAS2/ SAS3 was $85.5 \% / 50.0 \% / 24.5 \%$ that of plant growing in SCK (Fig. $4 a$ ). Accumulation amount of NP in stem of plant growing in SAS1/SAS2/ SAS3 was $82.9 \% / 49.5 \% / 23.8 \%$ that of plant growing in control soil while accumulation amount of NP in leaf of plant growing in SAS1/ SAS2/SAS3 was $81.5 \% / 53.1 \% / 25.2 \%$ that of plant growing in SCK (Fig. 4b). Accumulation amount of BPA in leaf/stem reached the highest with 621.93/60.04 ng for M. crystallinum growing in control soil while that of NP in leaf/stem reached the highest with 429.63/ $71.12 \mathrm{ng}$ in leaf/stem of plant growing in SCK. Accumulation amount of BPA in leaf accounted for $59.4 \%-78.6 \%$ of total accumulated BPA in whole plant while that of NP in root covered $68.5 \%$ $85.1 \%$ of total accumulated NP in whole plant. Significant difference in accumulated amount of BPA and NP in different parts of M. crystallinum might be caused by chemical properties of target EDCs and features of the plant. Higher salinity significantly decreased the accumulation amount of the target EDCs in M. crystallinum as well as the plant yield. Therefore, selection of suitable saline soil might directly affect the cleaner production of using reclaimed water irrigation. Soils like SAS1 with low salinity might be more suitable for cultivating $M$. crystallinum using reclaimed water irrigation by considering the accumulation of pollutant and plant yield. Reclaimed water irrigation in low-salinity soil might be an effective pathway for cleaner production of vegetables and other crops. Mechanisms such as the development and effect of plant bladder cells on BPA/NP accumulation in $M$. crystallinum under different soil salinities with reclaimed water irrigation might be needed for the future study. 

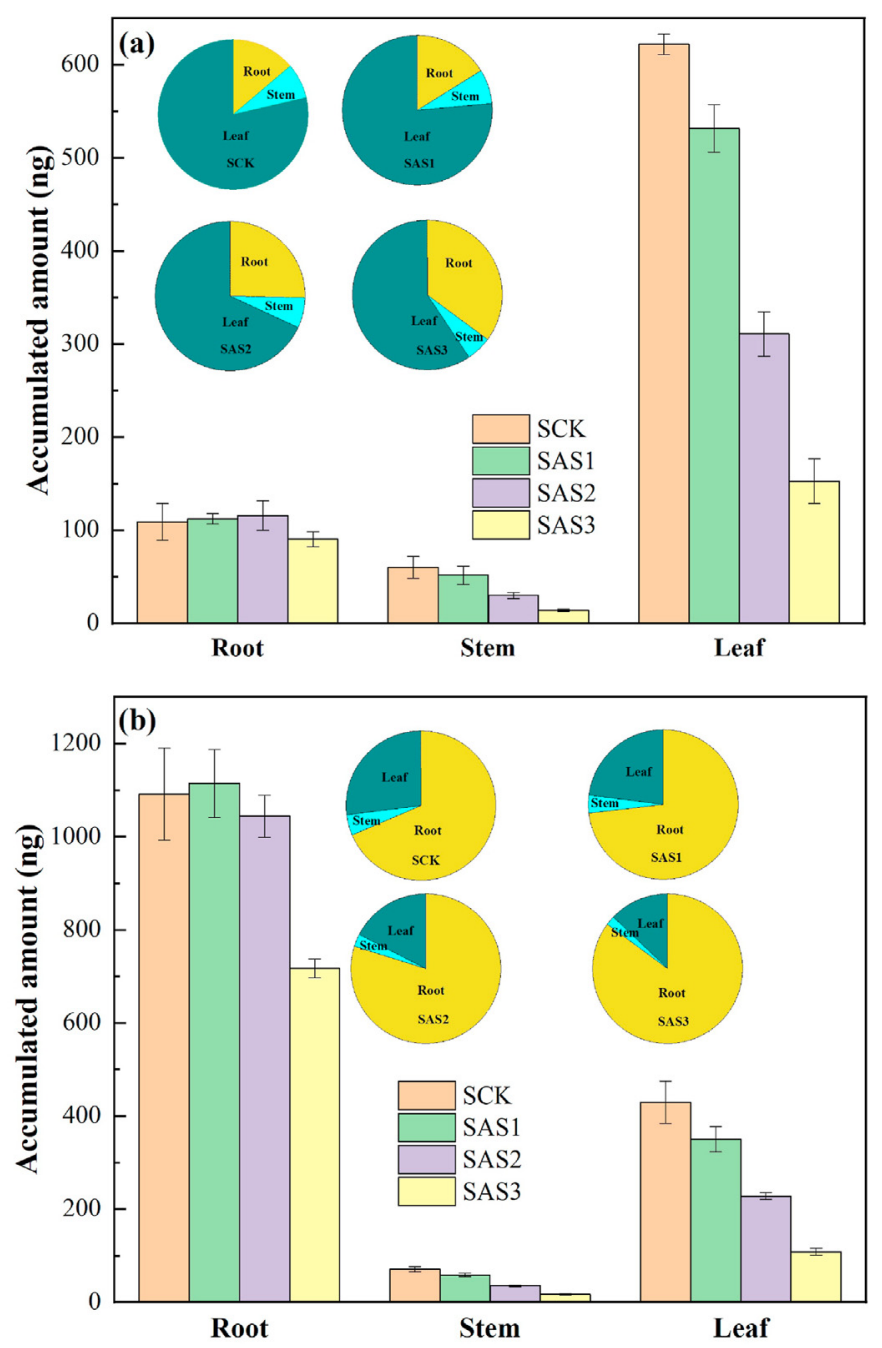

Fig. 4. Accumulation of BPA (a) and NP (b) in different parts of M. crystallinum. SCK refers to control soil; SAS1/SAS2/SAS3 refers to salt-affected soil with salinity of $1.1 \%$ / $2.4 \% / 7.2 \%$.

\subsection{Translocation of BPA and NP in M. crystallinum as well as the tolerance of plant}

The translocation of BPA and NP in M. crystallinum showed difference (Fig. 5). Translocation factors from root to stem were the lowest while those from stem to leaf were the highest for BPA and NP. Translocation factors from stem to leaf reached 6.35 for BPA and 3.67 for NP (Fig. 5a), exhibiting that BPA and NP were more readily to transport to leaf. Translocation factors of BPA decreased with increased salt content in soils while those from root to stem or leaf decreased to higher extent, which might be ascribed to the increased concentration of BPA in root. Compared with BPA, very low translocation factors from root to stem or leaf of NP caused more accumulated amount of NP in root of M. crystallinum (Fig. 5b). Pollutants generally move in the root by following the order of root hairs $\rightarrow$ intracellular spaces $\rightarrow$ cortical cell walls $\rightarrow$ endodermis $\rightarrow$ xylem and organic pollutants with log-based octanol-water partition coefficient $\left(K_{o w}\right)$ greater than 3.5 can be easily absorbed in soil granule and root surface of the plants (Kvesitadze et al., 2015). The $\log K_{o w}$ value of BPA/NP was 3.40/4.48 (Lu et al., 2015) to suggest that the target EDCs could be easily accumulated in the root part. $K_{o w}$ also has significant effect on transport of non-ionized organic pollutants from root to shoot and $K_{o w}$ of 1.8 or less is optimal for
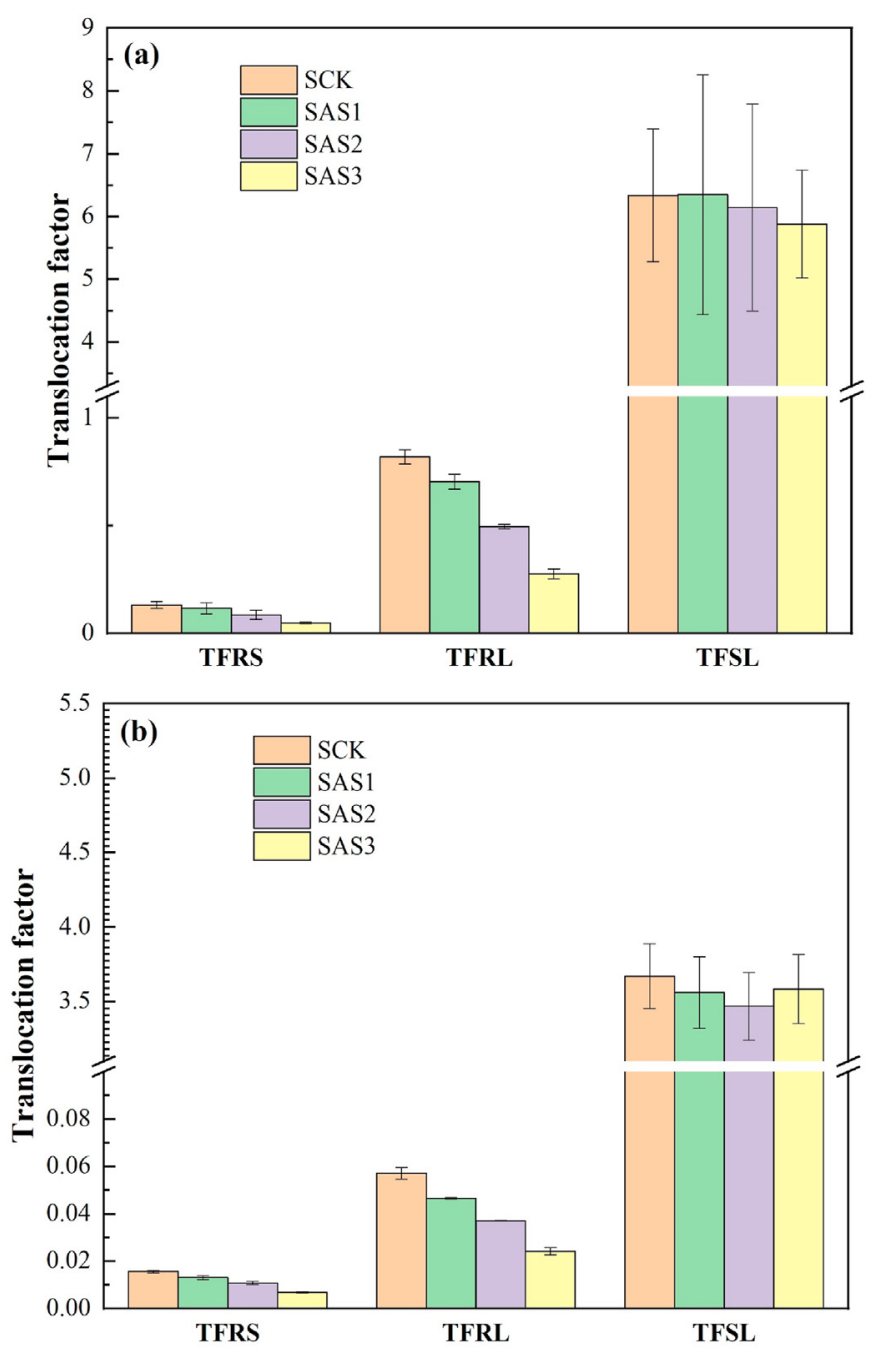

Fig. 5. Translocation factor of BPA (a) and NP (b) in M. crystallinum. SCK refers to control soil; SAS1/SAS2/SAS3 refers to salt-affected soil with salinity of $1.1 \% / 2.4 \%$ o/ 7.2\%; TFRS represents translocation from root to stem; TFRL represents translocation from root to leaf; TFSL represents translocation from stem to leaf.

pollutant to move across the membranes (Kvesitadze et al., 2015). It is obvious that translocation from root to shoot is not easy for BPA and NP in the plants due to their high $K_{o w}$ values.

Tolerance of M. crystallinum to BPA and NP was similar (Fig. 6). Interestingly, M. crystallinum showed good tolerance to target EDCs with all tolerance indices greater than 1, illustrating that addition of EDCs might serve as the hormone which could be helpful to alleviate the salt stress on the plant growth. Previous investigations have proved that EDCs could promote the growth of crops (Pan et al., 2013).

\subsection{Health risk assessment of BPA and NP in M. crystallinum}

Non-cancer health risks of BPA and NP in M. crystallinum were caused by oral ingestion (Fig. 7). The evaluation was performed by concentrations of BPA and NP in leaf of M. crystallinum. HQs of BPA and NP in M. crystallinum in different soils for all human groups followed the order of SCK $>$ SAS1 $>$ SAS2 $>$ SAS3. The HQs sharply decreased by more than $50 \%$ when the soil salinity increased from $1.1 \%$ to $7.2 \%$. Concentrations of BPA and NP in leaf of $M$. crystallinum growing in soils decreased with the soil salt content, which could be helpful to decrease the health risks. It should 

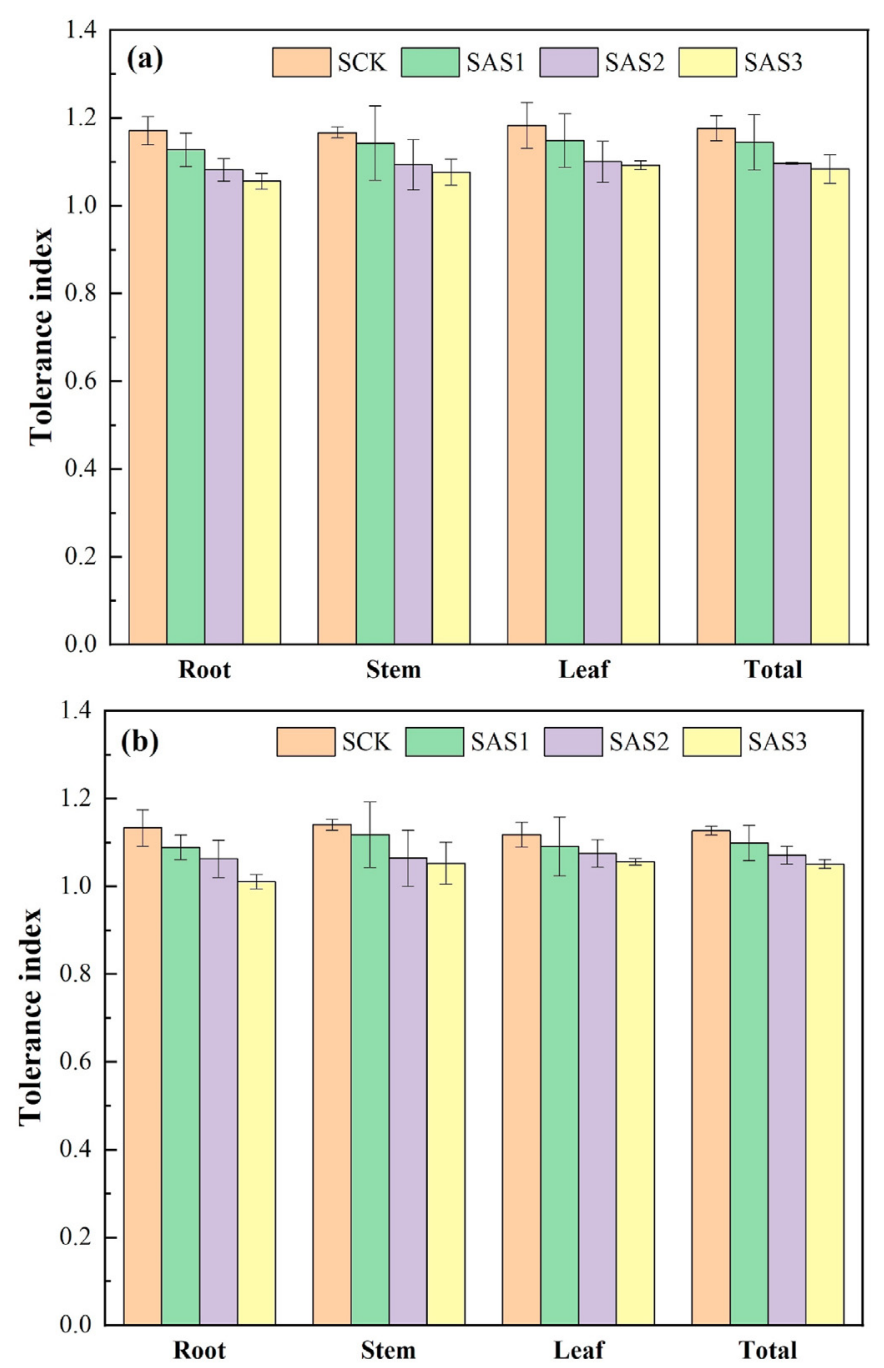

Fig. 6. Tolerance index of BPA (a) and NP (b) in different parts of M. crystallinum. SCK refers to control soil; SAS1/SAS2/SAS3 refers to salt-affected soil with salinity of $1.1 \%$ o/ $2.4 \% 0 / 7.2 \%$.

be paid attention to that the health risk of BPA/NP for children was almost 1.9-2.4 times that for teenagers and adults. Hazard quotients of BPA and NP for females were higher than those for males of the same age group, which might be influenced relatively high daily intake amount of $M$. crystallinum and lower weight of female groups. Compared with BPA, HQs posed by NP in M. crystallinum were much lower due to higher RfD value. All HQs posed by BPA and NP for different human groups were much lower than 1.0 to show acceptable risk, illustrating that it might be safe to daily consume M. crystallinum.

Daily intake of BPA through eating $M$. crystallinum was in the range of $(1.87-4.04) /(2.36-5.09) /(1.99-4.28) /(2.79-6.00) /$ (2.89-6.22) $\mu \mathrm{g} / \mathrm{d}$ for children/female teenagers/male teenagers/ female adults/male adults while daily intake of NP was in the range of $\quad(1.36-2.78) /(1.72-3.51) /(1.45-2.95) /(2.03-4.14) /(2.10-4.29)$ $\mu \mathrm{g} / \mathrm{d}$ for the corresponding group. The tolerance daily intake (TDI) of BPA and NP was calculated based on the recommended threshold by the European Commission Scientific Committee on Food and the Danish Institute of Safety and Toxicology, respectively (Lu et al., 2015). The TDI of BPA/NP for children, female teenagers, male teenagers, female adults, and male adults were 19.7/9.9, 47.6/23.8, $51.2 / 25.6,55.2 / 27.6$, and $63.3 / 31.6 \mu \mathrm{g} / \mathrm{d}$, respectively. All daily intake amounts of BPA/NP were much lower than the corresponding TDIs, also illustrating that consumption of $M$. crystallinum was safe. Higher soil salinity decreased the concentration and accumulation amount of BPA and NP in M. crystallinum to further reduce the health risks of these harmful chemicals under the conditions of reclaimed water irrigation. Li et al. (2010) also remarked that soil salinity might be useful to reduce the health risk of heavy metals in fruits to further support the results of this study. Consumption of $M$. crystallinum growing in saline soils and cultivated by reclaimed water would decrease daily intake of target EDCs, exhibiting that plant cultivation in saline soils coupled with reclaimed water irrigation might be a promising approach for utilization of coastal saline soils.

Cleaner production means less pollutant accumulation and lower health risks for vegetable cultivation. Salinity decreased concentration and accumulation of BPA and NP in M. crystallinum to further alleviate the potential health risks posed by these pollutants, illustrating that vegetable cultivation in saline soils with reclaimed water irrigation could be a useful trial for crop cleaner production.

\subsection{Life cycle assessment of M. crystallinum cultivation in salt- affected soils irrigated by reclaimed water}

LCA using five indices was performed to explore whether M. crystallinum cultivation in coastal saline soils irrigated by reclaimed water would cause significantly increased environmental burden (Fig. 8). Acidification/eutrophication of M. crystallinum growing in SAS2 and SAS3 was only $9.3 \%$ and $11.1 \%$ higher than that in SCK soil. The remaining indices including climate change, ecotoxicity, fossil fuels, and land use of M. crystallinum cultivation in SAS2 and SAS3 were only $9.0 \%-9.4 \%$ and $11.0 \%-12.1 \%$ higher than those of the plant growing in control soil. It was interesting that all indices except land use of M. crystallinum growing in SAS1 with salt content of $1.1 \%$ o were significantly lower than those of the plant growing in other soils. Climate change, ecotoxicity, fossil fuels, and acidification/eutrophication of M. crystallinum growing in SAS1 were only 3.3\%, $11.4 \%$, $23.8 \%$, and $2.2 \%$ of that of the plant growing in control soil. Land use of the ice plant (M. crystallinum) growing in SAS1 were 1.9/1.7/1.7 times that of the plant growing in SCK/SAS2/SAS3.

The yield of $M$. crystallinum growing in SAS1 and SAS2 was comparable with that in control soils. Low concentration and accumulation amount of the target EDCs in M. crystallinum growing in SAS1 and SAS2 showed the potential of cultivation of vegetables in saline soils. Low environmental burden of M. crystallinum cultivation in SAS1 approved that reclaimed water irrigation in coastal saline soils with low salt content could be a perspective method for coastal saline soil utilization. Coastal areas possess wide saline soils, a large population, and fast-developing economy (Wang et al., 2020). Water scarcity has become an important problem in the world (UN, 2019), especially the coastal areas. Reclaimed water usage will become an important strategy for humans to alleviate the water scarcity. LCA results of this study further confirmed the feasibility and effectiveness of cultivating $M$. crystallinum in slight saline soil irrigated by reclaimed water, which also showed that reclaimed water irrigation could be useful for the cleaner crop production in coastal saline soils.

\section{Conclusions}

It is necessary to discuss the effect of reclaimed water irrigation on cleaner vegetable production in saline soils since the previous studies seldom mentioned that. This study firstly reported that soil salinity decreased the accumulation of BPA and NP in 

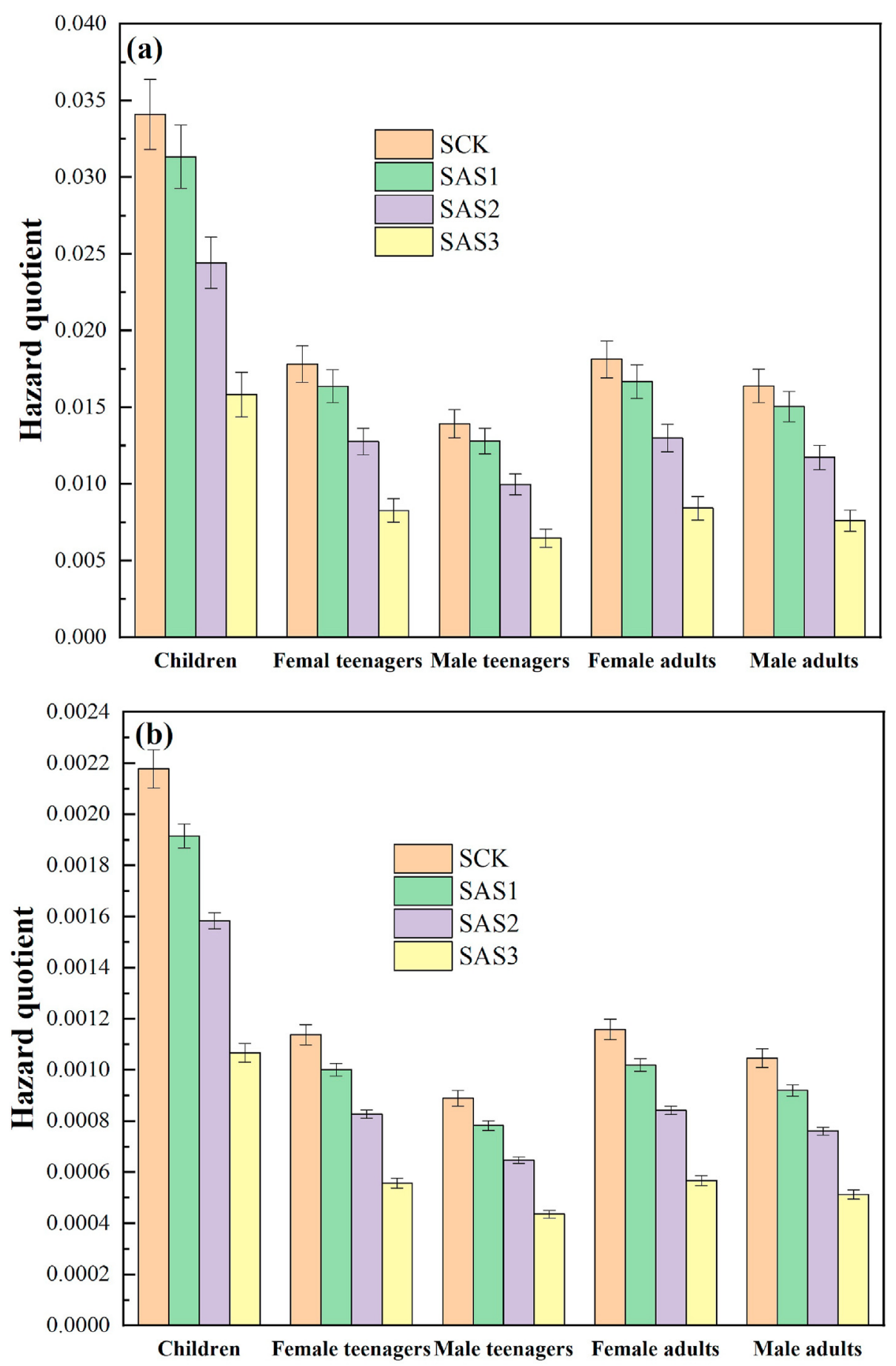

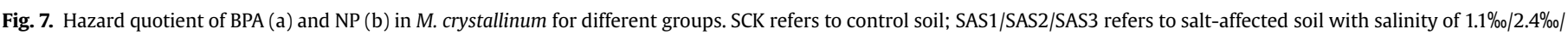
$7.2 \%$.

M. crystallinum to alleviate the potential health risks by $8.1 \%-53.6 \%$ for BPA and $12.1 \%-51.0 \%$ for NP. Concentrations of BPA and NP in root were higher than those in stem and leaf while accumulated amount of $\mathrm{BPA} / \mathrm{NP}$ in leaf/root covered $(59.4 \%-78.6 \%) /(68.5 \%-$ $85.1 \%$ ) of total accumulated target EDC in M. crystallinum. It was safe to daily consume $M$. crystallinum due to acceptable health risks posed by target EDCs in it. The M. crystallinum cultivation in slight saline soil irrigated by reclaimed water posed low environmental burden and low accumulation of EDCs. These results suggested that cultivating crops in the coastal saline soils with reclaimed water irrigation might be a good trial for the cleaner crop production in terms of saving water resource, utilizing saline soils, and alleviating the potential risks. These findings provide useful evidence that crop production irrigated with reclaimed water will be the promising practice for the coastal saline soil utilization. Cultivation strategies and optimal operation conditions of salt-tolerance plants in saline soils are recommended for future works. 

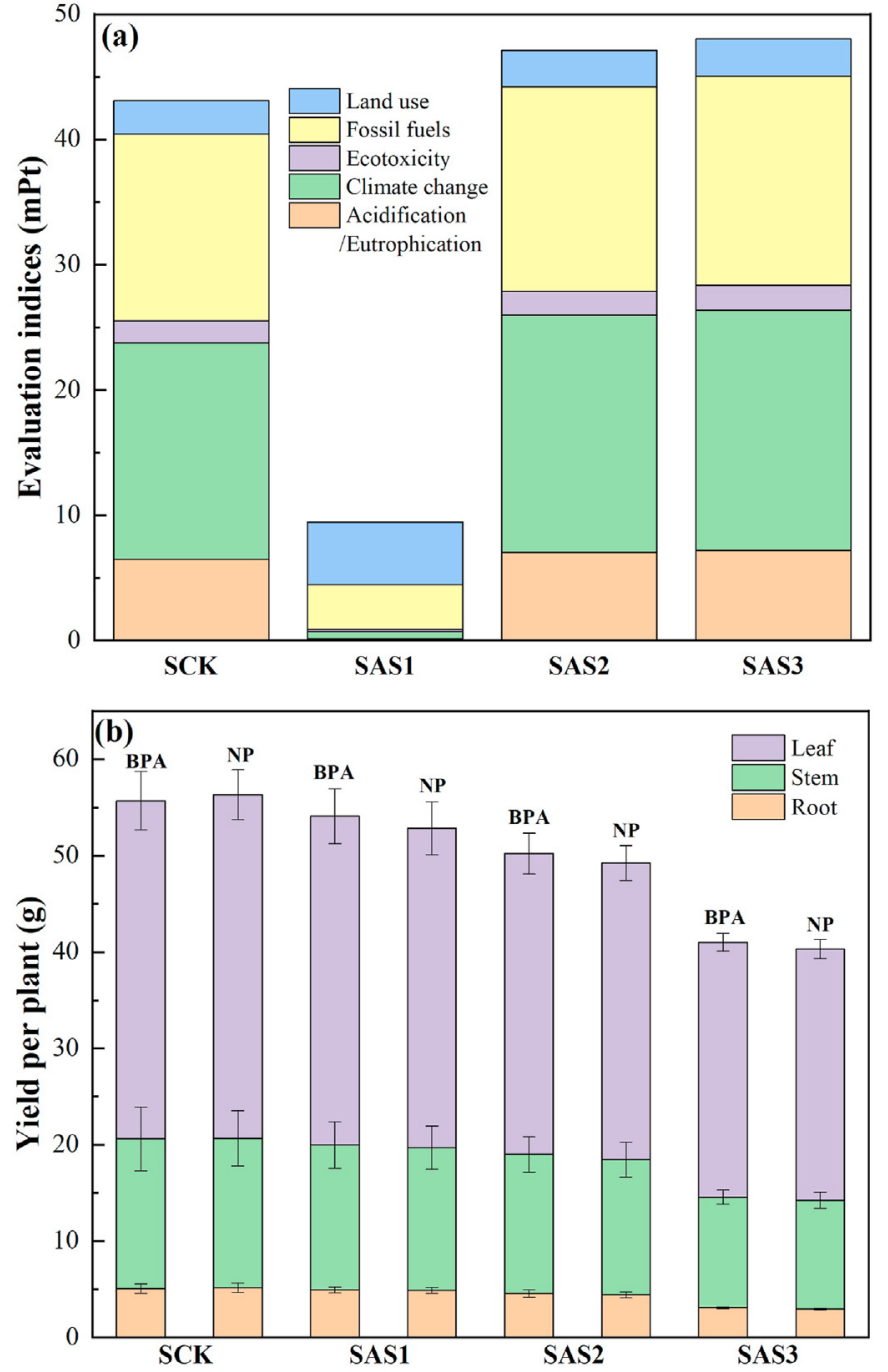

Fig. 8. Life cycle assessment results for crop cultivation in common soil and saline soils. SCK refers to control soil; SAS1/SAS2/SAS3 refers to salt-affected soil with salinity of $1.1 \% 0 / 2.4 \% 0 / 7.2 \%$.

\section{CRediT authorship contribution statement}

Jian Lu: Conceptualization, Funding acquisition, Investigation, Methodology, Project administration, Writing - original draft, Writing - review \& editing. Jun Wu: Conceptualization, Methodology, Investigation, Software, Supervision, Writing - original draft, Writing - review \& editing. Cui Zhang: Formal analysis, Investigation, Methodology.

\section{Declaration of competing interest}

The authors declare that they have no known competing financial interests or personal relationships that could have appeared to influence the work reported in this paper.

\section{Acknowledgements}

This work was financially supported by National Natural Science Foundation of China (41671319), Taishan Scholars Program of Shandong Province (tsqn201812116), Science and Technology Service Network Initiative of the Chinese Academy of Sciences (KFJ-
STS-QYZX-114), and Two-Hundred Talents Plan of Yantai (No. Y739011021). We also would like to thank the reviewers for their valuable comments and suggestions on the manuscript.

\section{Abbreviations in this paper}

BPA bisphenol A

NP nonylphenol

M. crystallinum Mesembryanthemum crystallinum

EDCs endocrine disrupting chemicals

LCA Life cycle assessment

LCI life cycle inventories

TMCS trimethylchlorosilane

BSTFA (N,O-bis(trimethylsilyl) trifluoroacetamide)

IDS isotope dilution standard

SAS1/SAS2/SAS3 soil with salinity of $1.1 \%$ / $2.4 \%$ o $7.2 \%$

SCK control soil sample

PCA principal component analysis

HQ hazard quotients

$R f D$ the reference dose through oral exposure route

AT average lifespan

$B W$ body weight

IR vegetable consumption rate

$E D \quad$ ingestion exposure duration

$E F \quad$ ingestion exposure frequency

$K_{o w} \quad$ octanol-water partition coefficient

TDI tolerance daily intake

TFRS translocation from root to stem

TFRL translocation from root to leaf

TFSL translocation from stem to leaf

SC soil salinity

EC electrical conductivity

CEC cation exchange capacity

BPAR BPA in root

BPAL BPA in leaf

BPAS BPA in stem

NPR NP in root

NPL NP in leaf

NPS NP in stem

\section{References}

Ahmadisharaf, E., Benham, B.L., 2020. Risk-based decision making to evaluate pollutant reduction scenarios. Sci. Total Environ. 702, 135022.

Athar, M., Zaidi, S., 2020. A review of the feedstocks, catalysts, and intensification techniques for sustainable biodiesel production. J. Environ. Chem. Eng. 8, 104523.

Atzori, G., de Vos, A.C., van Rijsselberghe, M., Vignolini, P., Rozema, J., Mancuso, S., van Bodegom, P.M., 2017. Effects of increased seawater salinity irrigation on growth and quality of the edible halophyte Mesembryanthemum crystallinum L. under field conditions. Agric. Water Manag. 187, 37-46.

Cascone, S., Ingrao, C., Valenti, F., Porto, S.M.C., 2020. Energy and environmental assessment of plastic granule production from recycled greenhouse covering films in a circular economy perspective. J. Environ. Manag. 254, 109796.

Chiconato, D.A., da Silveira Souza Junior, G., dos Santos, D.M.M., Munns, R., 2019. Adaptation of sugarcane plants to saline soil. Environ. Exp. Bot. 162, 201-211.

Dodgen, L.K., Li, J., Parker, D., Gan, J.J., 2013. Uptake and accumulation of four PPCP/ EDCs in two leafy vegetables. Environ. Pollut. 182, 150-156.

Fritiof, A., Kautsky, L., Greger, M., 2005. Influence of temperature and salinity on heavy metal uptake by submersed plants. Environ. Pollut. 133, 265-274.

Futran Fuhrman, V., Tal, A., Arnon, S., 2015. Why endocrine disrupting chemicals (EDCs) challenge traditional risk assessment and how to respond. J. Hazard Mater. 286, 589-611.

Giulivo, M., Lopez de Alda, M., Capri, E., Barceló, D., 2016. Human exposure to endocrine disrupting compounds: their role in reproductive systems, metabolic syndrome and breast cancer. A review. Environ. Res. 151, 251-264.

Guerra, B., Leite, F., Faust, K.M., 2020. 4D-BIM to enhance construction waste reuse and recycle planning: case studies on concrete and drywall waste streams. Waste Manag. 116, 79-90.

Gunady, M.G.A., Biswas, W., Solah, V.A., James, A.P., 2012. Evaluating the global warming potential of the fresh produce supply chain for strawberries, romaine/ cos lettuces (Lactuca sativa), and button mushrooms (Agaricus bisporus) in 
Western Australia using life cycle assessment (LCA). J. Clean. Prod. 28, 81-87. Hermabessiere, L, Dehaut, A., Paul-Pont, I., Lacroix, C., Jezequel, P., Soudant, P., Duflos, G., 2017. Occurrence and effects of plastic additives on marine environments and organisms: a review. Chemosphere 182, 781-793.

Kvesitadze, G., Khatisashvili, G., Sadunishvili, T., Kvesitadze, E., 2015. Plants for remediation: uptake, translocation and transformation of organic pollutants. In: Öztürk, M., Ashraf, M., Aksoy, A., Ahmad, M.S.A., Hakeem, K.R. (Eds.), Plants, Pollutants and Remediation. Springer Netherlands, USA, pp. 241-305.

Li, Q., Cai, S., Mo, C., Chu, B., Peng, L., Yang, F., 2010. Toxic effects of heavy metals and their accumulation in vegetables grown in a saline soil. Ecotoxicol. Environ. Saf. $73,84-88$

Li, Y.C., Jiang, B., Zhang, M., Huang, Z.J., Deng, Q., Zhou, M.G., Zhao, Z.P., Wang, Y.F., Wang, L.M., 2017. Vegetable and fruit consumption among Chinese adults and associated factors: a nationally representative study of 170,847 adults. Biomed. Environ. Sci. 30, 863-874.

Lu, J., Wu, J., Stoffella, P.J., Wilson, P.C., 2015. Uptake and distribution of bisphenol A and nonylphenol in vegetable crops irrigated with reclaimed water. J. Hazard Mater. 283, 865-870.

Lu, J., Wu, J., Zhang, C., Zhang, Y., 2020a. Possible effect of submarine groundwater discharge on the pollution of coastal water: occurrence, source, and risks of endocrine disrupting chemicals in coastal groundwater and adjacent seawater influenced by reclaimed water irrigation. Chemosphere 250, 126323.

Lu, J., Zhang, C., Wu, J., Zhang, Y., Lin, Y., 2020b. Seasonal distribution, risks, and sources of endocrine disrupting chemicals in coastal waters: will these emerging contaminants pose potential risks in marine environment at continental-scale? Chemosphere 247, 125907.

Minhas, P.S., Ramos, T.B., Ben-Gal, A., Pereira, L.S., 2020. Coping with salinity in irrigated agriculture: crop evapotranspiration and water management issues. Agric. Water Manag. 227, 105832.

Pan, W.-J., Xiong, C., Wu, Q.-P., Liu, J.-X., Liao, H.-M., Chen, W., Liu, Y.-S., Zheng, L., 2013. Effect of BPA on the germination, root development, seedling growth and leaf differentiation under different light conditions in Arabidopsis thaliana.
Chemosphere 93, 2585-2592.

Rosário, R., Santos, R., Lopes, L., Agostinis-Sobrinho, C., Moreira, C., Mota, J. Póvoas, S., Oliveira, A., Padrão, P., Moreira, P., Abreu, S., 2018. Fruit, vegetable consumption and blood pressure in healthy adolescents: a longitudinal analysis from the LabMed study. Nutr. Metab. Cardiovas. 28, 1075-1080.

Santonicola, S., Ferrante, M.C., Murru, N., Gallo, P., Mercogliano, R., 2020. Hot topic: bisphenol A in cow milk and dietary exposure at the farm level. J. Dairy Sci. 102, 1007-1013.

Santos, T.L., Nunes, A.B.A., Giongo, V., Barros, V.S., de Figueirêdo, M.C.B., 2018. Cleaner fruit production with green manure: the case of Brazilian melons. J. Clean. Prod. 181, 260-270.

Taghipour, M., Jalali, M., 2019. Impact of some industrial solid wastes on the growth and heavy metal uptake of cucumber (Cucumis sativus L.) under salinity stress. Ecotoxicol. Environ. Saf. 182, 109347.

UN (United Union), 2019. World Water Development Report (WWDR 2019): Leaving No One behind.

Van Loy, S., Binnemans, K., Van Gerven, T., 2018. Mechanochemical-assisted leaching of lamp phosphors: a green engineering approach for rare-earth recovery. Engineering 4, 398-405.

Wang, J., Yuan, G., Lu, J., Wu, J., Wei, J., 2020. Variation of salts and available nutrients in salt-affected soil during leaching process under the influence of organic ameliorators. Chem. Ecol. 36, 256-269.

Wen, X., Lu, J., Wu, J., Lin, Y., Luo, Y., 2019. Influence of coastal groundwater salinization on the distribution and risks of heavy metals. Sci. Total Environ. 652, 267-277.

Zalacáin, D., Martínez-Pérez, S., Bienes, R., García-Díaz, A., Sastre-Merlín, A., 2019. Salt accumulation in soils and plants under reclaimed water irrigation in urban parks of Madrid (Spain). Agric. Water Manag. 213, 468-476.

Zheng, N., Wang, Q., Zheng, D., 2007. Health risk of Hg, Pb, Cd, Zn, and $\mathrm{Cu}$ to the inhabitants around Huludao Zinc Plant in China via consumption of vegetables. Sci. Total Environ. 383, 81-89. 